\title{
A numerical procedure for minimizing the maximum cost
}

\author{
Silvia Di Marco, Roberto L.V. González \\ Universidad Nacional de Rosario
}

Fac. Cs. Ex., Ing. y Agrim., Universidad Nacional de Rosario,

Avda. Pellegrini 250, (2000) Rosario, Argentina.

Fax: (54) 41 257164. e-mail: Dimarco@bibfei.edu.ar

\begin{abstract}
In this paper we consider the numerical solution of a minimax optimal control problem, where the cost to be minimized is the maximum of a function which depends on the state and the control. We present an approximation method which employs both discretization on time and on spatial variables. In this way, we obtain a computational implementable fully discrete problem. We give an optimal estimate for the error between the approximated solution and the optimal cost of the original problem.
\end{abstract}

\section{Keywords}

Minimax problems, numerical solution, quasi-variational inequalities

\section{INTRODUCTION AND DESCRIPTION OF THE PROBLEM}

We consider in the interval $[0, T]$ a dynamic system which evolves according to the ordinary differential equation

$$
\mid \begin{array}{ll}
\frac{d y}{d s}(s)=g(y(s), \alpha(s)) & 0 \leq t \leq s \leq T, \\
(t)=x \in \Omega \subseteq \mathrm{R}^{r}, & \Omega \text { an open domain. }
\end{array}
$$

The optimal control problem consists in minimizing the functional $J$

$$
J:(t, x, \alpha(\cdot)) \in[0, T] \times \Omega \times \mathcal{U}(t, T) \mapsto \operatorname{ess} \sup \{f(y(s), \alpha(s)): s \in[t, T)\} .
$$

The set of controls is $\mathcal{U}(t, s)=\left\{\alpha:[t, s] \rightarrow A \subset \mathrm{R}^{m}: \alpha(\cdot)\right.$ measurable $\}$.

This problem arises, for example, when we want to minimize the maximum deviation of the controlled trajectories with respect to a given special trajectory. This differs from those problems usually considered in the optimal control literature, where an accumulated 
cost is minimized. As considering an accumulated cost is not always the best method to qualify a controlled system with an unique scalar parameter, problems of this type has received considerable interest in recent publications (see e.g. (Barron and Ishii, 1989)).

The objective of this work is to approximate in a numerical way the value function $u$

$u:(t, x) \in[0, T] \times \Omega \mapsto \inf \{J(t, x, \alpha(\cdot)): \alpha \in \mathcal{U}(t, T)\}$.

We assume that $f$ and $g$ are bounded and uniformly continuous functions on $\Omega \times A$, $A$ is compact and the trajectory $y(\cdot)$ remains in $\Omega$, for any control belonging to $\mathcal{U}(t, T)$. In that case, the function $\mathrm{u}$ has the following properties, which have been established by (Barron and Ishii, 1989):

- $u$ is a bounded and uniformly continuous function and it is also Lipschitz continuous in its second variable.

- $u$ satisfies the following dynamic programming principle $\forall t \in[0, T), x \in \Omega, s<t$,

$u(t, x)=\inf \left\{\max \left\{\operatorname{esssup}_{\tau \in[t, s]} f(y(\tau), \alpha(\tau)), u(s, y(s))\right\}: \alpha \in \mathcal{U}(t, s), s \in(t, T]\right\}$,

with final condition

$u(T, x)=\min \{f(x, a): a \in A\}$.

The principal results obtained in our work are the following ones:

1. We obtain a discrete time approximation using a finite differences scheme and we give an estimate of the error of this approximation.

2. By using linear finite elements, we obtain a fully discrete approximation that converges to the solution of the original problem with rate $\sqrt{k}$.

3. We show the optimality of the estimation $\sqrt{k}$.

\section{A DISCRETE TIME SCHEME OF APPROXIMATION}

Following the methodology used in (González and Tidball, 1992), we define an auxiliary problem which is a natural discretization of the optimal cost $u$ defined in (3). We divide the interval $[0, T]$ into $\mu$ subintervals with common length $h=T / \mu$. We define recursively, $\forall n=0, \ldots, \mu-1, x \in \Omega$.

$u^{h}(n, x)=\min _{a \in A}\left\{\max \left\{f(x, a), u^{h}(n+1, x+h g(x, a))\right\}\right\}$,

with the final condition

$u^{h}(\mu, x)=\min _{a \in A} f(x, a)$.

The function $u^{h}$ can be interpreted as the optimal cost function of a discrete time optimal control problem, which is a natural discretization of problem (3). From (4) and 
(5), it is easy to prove that $u^{h}$ is bounded and Lispschitz continuous. The function $u^{h}$ approximates the function $u$ with a rate of convergence given by the following theorem.

Theorem 1 Let $u(t, x)$ be the optimal cost of the original problem and $u^{h}(n, x)$ the discrete time cost defined in (4) - (5), then the following estimate of the difference between $u^{h}$ and $u$ holds

$\left|u(n, x)-u^{h}(n, x)\right| \leq M \sqrt{h}$.

\section{$3 \quad$ FULLY DISCRETE SOLUTIONS}

With the previous scheme we have approximated the function $u$ with one obtained by discretizing the original problem in its time variable. This approximation scheme is not directly implementable to be computed numerically. To obtain a fully discrete approximation with this property, we discretize the space $\Omega$, using the methodology described in (González and Rofman, 1985) or (González and Tidball, 1992)

\subsection{A fully discrete scheme of approximation}

We identify the discretization of the spatial variables with the parameter $k$, which also indicates the size of the discretization. We consider a family of quasi-uniform triangulations of $\Omega$; i.e. a family of polyhedrons $\Omega_{k}$ given by a finite collection of closed simplices $\left\{S_{j}^{k}\right\}$, such that $\Omega_{k}$ converges to $\Omega$ as $k$ goes to 0 .

Let $V_{k}=\left\{x^{i}, i=1, \ldots, N\right\}$ be the vertices of $\Omega_{k}$, arbitrarily arranged and $N$ its cardinal. Every $x \in \Omega_{k}$ is a convex combination of the vertices $x^{i}$ of the simplex to which $x$ belongs. Hence, $\forall a \in A$ there exists a matrix $N \times N$, with components $\gamma_{j}\left(x^{i}, a\right)$, such that:

$\gamma_{j}\left(x^{i}, a\right) \geq 0, \sum_{j=1}^{N} \gamma_{j}\left(x^{i}, a\right)=1, x^{i}+h g\left(x^{i}, a\right)=\sum_{j=1}^{N} \gamma_{j}\left(x^{i}, a\right) x^{j}$.

We consider the set $W_{k}$ of functions $w: \Omega_{k} \rightarrow \mathbf{R}, w \in W^{1, \infty}\left(\Omega_{k}\right)$, such that $\partial w / \partial x$ is constant in the interior of each simplex of $\Omega_{k}$, i.e., the functions $w$ are linear finite elements and they are characterized by their values on $V_{k}$. We denote $F_{k}=\left(W_{k}\right)^{\mu+1}$ and the elements of $F_{k}$ will be denoted $w_{k}^{h}(n, x), n=0, \ldots, \mu, x \in V_{k}$.

Taking in mind the equation (4)-(5), we define the fully discrete solution to be the function $u_{k}^{h} \in F_{k}$ such that, $\forall x^{i} \in V_{k}, u_{k}^{h}\left(\mu, x^{i}\right)=\min _{a \in A} f\left(x^{i}, a\right)$ and, $\forall n=0, \ldots, \mu-1$, it verifies the relation

$u_{k}^{h}\left(n, x^{i}\right)=\min _{a \in A}\left\{\max \left\{f\left(x^{i}, a\right), \sum_{j=1}^{N} \gamma_{j}\left(x^{i}, a\right) u_{k}^{h}\left(n+1, x^{j}\right)\right\}\right\}$.

Obviously, the solutions of these equations are unique and can be computed recursively. This allows us to implement the computational procedure. 


\subsection{Central result: Rate of convergence}

The central result of convergence is given by the following theorem which establishes an estimate of the difference between the optimal cost and the fully discrete solution. The proof is based on regularization techniques; essentially, it consists in obtaining estimates for the differences between subsolutions and supersolutions of problems introduced ad-hoc.

Theorem 2 If there exist constants $c_{1}$ and $c_{2}$ such that $c_{1} k \leq h \leq c_{2} k$, then there exists $C$ such that $\forall x \in V_{k}, \forall n=0, \ldots, \mu$, it results

$\left|u(n h, x)-u_{k}^{h}(n, x)\right| \leq C \sqrt{k}$.

Note 1 Some computational applications of our discretization procedure have been presented in detail in (Di Marco and González, 1995a).

\section{OPTIMALITY OF THE ESTIMATE}

In this problem, even though the data $f$ and $g$ are semiconcave in $x$, it is not possible to improve the estimate $\sqrt{h}$ which appears in (6), as it was done in the problem studied in (González and Tidball, 1992). There, under semiconcavity hypotheses on $f$ and $g$, it was shown that the optimal cost function $u$ also results semiconcave. In that case, the estimate for $\left\|u-u^{h}\right\|$ can be improved to order $h$, improvement that was crucial to prove an estimate of type $k^{2 / 3}$ for the fully discrete approximation. The following example shows that, for the minimax problem, an improvement of this type is not possible.

Let the dynamic of the system be

$$
\left\{\begin{array}{l}
\frac{d y}{d t}(t)=\left(\begin{array}{cc}
0 & 1 \\
-1 & 0
\end{array}\right) y(t), \quad \forall t \in[0,10], \\
y(0)=x \in \mathbf{R}^{2}
\end{array}\right.
$$

We define the instantaneous cost function as follows

$f(y(t))=\left(1-\left(y_{1}^{2}(t)+y_{2}^{2}(t)\right)\right) y_{1}(t)$

where $y_{i}(\cdot)$ is the $i$ th component of $y(\cdot)$.

The system moves freely in $\mathrm{R}^{2}$ and the functions $f$ and $g$ verify the assumed hypotheses. Clearly, they are semiconcave.

Let $r=\sqrt{x_{1}^{2}+x_{2}^{2}}$. It is easy to check, after elementary calculus that the optimal cost function is

$u(0, x)=\max _{t \in[0,10]} f(y(t))=\left|1-r^{2}\right| r$, 
which is not semiconcave.

The discretization procedure introduced here coincides with the methodology studied by (González and Tidball, 1992). In that work, the authors proved that the estimate $\sqrt{k}$ is critical. In fact, here we can prove - using a special triangulation and calculations entirely similar to those employed in (González and Tidball, 1992) - that the error $\mid u(0, x)-$ $u_{k}^{h}(0, x) \mid$ verifies

$\left|u(0, x)-u_{k}^{h}(0, x)\right| \geq C \sqrt{k}$.

In consequence, we have that for approximations of this type the better error that can be expected, is of order $\sqrt{k}$.

\section{GENERALIZATION TO ACCUMULATED COSTS}

Similarly to what we have done for function (2), we can deal with the general problem of minimizing a functional $\widehat{J}$ that includes an integral cost. Specifically, we consider the functional

$\widehat{J}(t, x, \alpha(\cdot))=\underset{s \in[t, T)}{\operatorname{esssup}}\left\{f(y(s), \alpha(s))+\int_{t}^{s} b(y(\theta), \alpha(\theta)) d \theta\right\}$,

where $y(\cdot)$ is the solution of the dynamical system (1) and the function $b$ is bounded and Lipschitz continuous in $x$. Here, the optimal

cost function $u$ takes the form

$u:(t, x) \in[0, T] \times \Omega \mapsto \inf \{\widehat{J}(t, x, \alpha(\cdot)): \alpha \in \mathcal{U}(t, T)\}$

Similarly to (8), we define recursively the fully discrete approximation of $u$ as follows,

$$
\begin{gathered}
\forall x^{i} \in V_{k}, u_{k}^{h}\left(\mu, x^{i}\right)=\min _{a \in A} f\left(x^{i}, a\right) \text { and, } \forall n=0, \ldots, \mu-1 \\
u_{k}^{h}\left(n, x^{i}\right)=\min _{a \in A}\left\{\max \left\{f\left(x^{i}, a\right), h b\left(x^{i}, a\right)+\sum_{j=1}^{\mu} \gamma_{j}\left(x^{i}, a\right) u_{k}^{h}\left(n+1, x^{j}\right)\right\}\right\} .
\end{gathered}
$$

where $\gamma_{j}\left(x^{i}, a\right)$ are the functions defined in (7).

Also for this problem, it holds the following convergence result:

Theorem 3 If there exist constants $c_{1}$ and $c_{2}$ such that $c_{1} k \leq h \leq c_{2} k$, then there exists a constant $C$ such that $\forall x \in V_{k}, \forall n=0, \ldots, \mu$

$\left|u(n h, x)-u_{k}^{h}(n, x)\right| \leq C \sqrt{k}$. 
Hence, for the general problem we can get fully discrete approximations and an estimate of convergence of the same order as that obtained for the original problem.

\section{FINAL COMMENTS}

Here, we have developed a discretization procedure to obtain the numerical solution of the problem of minimizing the maximum cost, analyzed from the continuous point of view by Barron and Ishii (see (Barron and Ishii, 1989)).

The numerical procedure obtained is easily implementable and it converges to the solution of the original problem, with an error estimate of the form

$\left|u-u_{k}^{h}\right| \leq C \sqrt{k}$.

This estimate was shown to be optimal.

The optimality of the estimate (17) stems from the fact that this minimax problem is a disguised differential game problem. In that game, the controller is trying to minimize the cost

$J(t, x, \alpha(\cdot), \tau)=f(y(\tau), \alpha(\tau))$,

while a hidden opponent - using full information of actions of the other player - chooses at any instant the stopping time $\tau$ of the process. The pay-off (18) is given as out-come of the complete game. As a result of the action of the second privileged player, the first player must - in a strict way - minimize a functional that is not semiconcave with respect to the spatial variable $y$. In this way, once a fully discrete approximation - using finite differences or finite elements - is applied, the resultant fully discrete optimal control problem reflects this property in the validity of the estimate of type $\sqrt{k}$.

Except for very special trajectories, where some carefully chosen triangulations may be used, it seems not possible in general to get approximations with better convergence properties.

In fact, the error worsens as time grows and for the case $T=\infty$, the phenomenon of non-convergence arises. This undesirable effect is studied more detailed in (Di Marco and González, 1995b).

\section{REFERENCES}

Barron, E.N. and Ishii, H. (1989) The Bellman equation for minimizing the maximum cost. Nonlinear Analysis, Theory, Methods \& Applications, 13, 1067-90.

Di Marco, S.C. and González, R.L.V. (1995a) A numerical procedure for minimizing the maximum cost. Rapport de Recherche $N^{\circ} 2454$, INRIA. 
Di Marco, S.C. and González, R.L.V. (1995b) A numerical procedure for minimizing the maximum cost - The infinite time problem. Work in progress.

González, R.L.V. and Rofman, E. (1985) On deterministic control problems: An Approximation procedure for the optimal cost, Parts 1 and 2. SIAM Journal on Control and Optimization, 23, 242-85.

González, R.L.V. and Tidball, M.M. (1992) Sur l'ordre de convergence des solutions discrétisées en temps et en espace de l'équation de Hamilton-Jacobi. Comptes Rendus Acad. Sci. Paris, Tome 314, Série I, 479-82. 